\title{
Análisis del contenido de información económico-técnica en las páginas web de empresas de transporte urbano en España
}

\author{
Cecilia Benítez-Quevedo**, Pedro M. Balboa-La Chica ${ }^{* * *}$, Margarita Mesa-Mendoza ${ }^{* * * *}$, \\ Heriberto Suárez-Falcón ${ }^{* * * * *}$
}

* Artículo de investigación

* Magíster en Contabilidad, Auditoría y Fiscalidad Empresarial, Universidad de Las Palmas de Gran Canaria. Correo electrónico: cecilia.benitez101@alu.ulpgc.es

*** Magíster en Contabilidad, Auditoría y Fiscalidad Empresarial, Universidad de Las Palmas de Gran Canaria. Correo electrónico: pedromanuel.balboa@ulpgc.es

**** Magíster en Contabilidad, Auditoría y Fiscalidad Empresarial, Universidad de Las Palmas de Gran Canaria. Correo electrónico: margarita.mesa@ulpgc.es

***** Departamento de Economía Financiera y Contabilidad, Universidad de Las Palmas de Gran Canaria.

Correo electrónico:

heriberto.suarez@ulpgc.es (autor de contacto)

Recibido: 3 de septiembre del 2016

Aprobado: 22 de enero del 2017

Cómo citar este artículo: Benítez-Quevedo C., Balboa-La Chica, P.M., Mesa- Mendoza, M. y Suárez-Falcón, H. (2017). Análisis del contenido de información económicotécnica en las páginas web de empresas de transporte urbano en España. Cooperativismo \& Desarrollo, 111(25), xx-xx. doi: https://doi. org/10.16925/co.v25i111.1773

\section{Resumen}

Propósito: en el presente artículo se analiza en qué medida las empresas prestatarias del transporte urbano de pasajeros en España publican en sus páginas web información de utilidad que permita evaluar la gestión realizada en un determinado periodo.Temas: se procede a la selección de la muestra de empresas a partir de aquellas que prestan el servicio en las capitales de provincias españolas, por ser los municipios de mayor relevancia y teniendo en cuenta su actividad fundamental y la disposición de sus páginas web. Desarrollo: a dicha muestra se aplica un cuestionario de diversos ítems, que presta especial atención a aspectos relativos a su organización, actividad, medios de producción técnicos, consumos, recursos humanos e información económica. Conclusiones: los resultados se presentan, tanto en función de la dimensión poblacional de las capitales de provincias, como en relación con la gestión directa o indirecta del servicio. Tales resultados ponen de manifiesto la escasa información de carácter económico, a excepción de las tarifas y el número de líneas. Además, las empresas de gestión directa aportan más información con respecto a aquellas que gestionan el servicio de forma indirecta.

Palabras clave: análisis de contenido, estrategia empresarial, responsabilidad social, transporte urbano. 


\title{
Content analysis of economic and technical information on Spain's urban transport companies' web pages
}

\begin{abstract}
Purpose: This article analyzes to what extent do companies that provide urban passenger transport in Spain publish useful information on their websites that allows evaluating the management carried out in a given period. Themes: The first step was to select the sample of companies from those providing the service in the capitals of Spanish provinces, since this are the most relevant municipalities and taking into account their fundamental activity and their web pages' layout. Development: A questionnaire of diverse items is then applied to the sample. The questionnaire emphasizes on aspects related to the companies' organization, activity, technical production means, consumptions, human resources and economic information. Conclusions: Results are presented, both in terms of the population size of provincial capitals and in relation to the direct or indirect management of the service. These results reveal the scarce economic information offered, with the exception of tariffs and the number of lines. In addition, it was found that companies with direct management provide more information than those that manage the service indirectly.
\end{abstract}

Keywords: content analysis, business strategy, social responsibility, urban transport

\section{Análise do conteúdo de informação econômico-técnica nas páginas web de empresas de transporte urbano na Espanha}

\section{Resumo}

Propósito: neste artigo, analisa-se em que medida as empresas prestadoras do transporte público urbano de passageiros na Espanha publicam em suas páginas web informação de utilidade que permita avaliar a gestão realizada num determinado período. Temas: procede-se à seleção da amostra de empresas a partir daquelas que prestam o serviço nas capitais de estados espanhóis, por serem os municípios de maior relevância e considerando sua atividade fundamental e a disposição de suas páginas web. Desenvolvimento: a essa amostra, aplica-se um questionário de diversos itens que presta especial atenção a aspectos relativos a sua organização, atividade, meios de produção técnicos, consumo, recursos humanos e informação econômica. Conclusões: os resultados são apresentados tanto em função da dimensão populacional das capitais quanto em relação à gestão direta ou indireta do serviço. Esses resultados manifestam a escassa informação de caráter econômico, à exceção das tarifas e do número de linhas. Além disso, as empresas de gestão direta contribuem com mais informação em comparação àquelas que administram o serviço de forma indireta.

Palavras-chave: análise de conteúdo, estratégia empresarial, responsabilidade social, transporte urbano. 


\section{Introducción}

El sector del transporte puede ser objeto de estudio desde distintas perspectivas: económica, social, jurídica, contable, financiera, etc. Todas ellas, sin duda, de notable interés. No podemos obviar que, tal y como señala la Comisión Europea (2011): “El transporte es fundamental para nuestra economía y nuestra sociedad. La movilidad es vital para el mercado interior y para la calidad de vida que aporta a los ciudadanos la libertad de viajar".

El objetivo de este trabajo consiste en analizar en qué medida las empresas prestatarias del servicio de transporte público de viajeros urbanos en España publican en sus páginas web información de utilidad para usuarios externos, con la intención de realizar un diagnóstico sobre la forma de organización, así como sobre los parámetros económicos y técnicos que constituyen indicadores de la gestión realizada en un determinado periodo.

Con tal fin, el trabajo se estructura en seis apartados. Después de esta introducción, pasaremos en el segundo apartado a contextualizar el transporte público urbano colectivo de viajeros en España. En el tercer apartado abordamos los aspectos metodológicos del estudio, lo que servirá de base para establecer en el siguiente los resultados. En el cuarto, presentamos los diferentes resultados atendiendo, en primer lugar, a las características globales que se obtienen de los datos $y$, en segundo lugar, a una segmentación que nos permita establecer conclusiones con mayor rigor y detalle. Finalmente, se cierra el trabajo con las principales conclusiones, además de las referencias bibliográficas.

Los resultados ponen de manifiesto la escasa información de índole económica y técnica que aportan las empresas en sus páginas web, a excepción de las tarifas y el número de líneas. No obstante, las empresas gestionadas de forma directa por la administración pública competente, proporcionan más información que en el caso de gestión indirecta del servicio. De otra parte, las empresas que prestan su servicio en las capitales de menor población son las que menos información publican en la web.

\section{El transporte público regular urbano de viajeros}

La movilidad se ha convertido en una necesidad básica, ya que nos permite acceder al trabajo, a la educación, a los hospitales, al ocio y a otros servicios. De esta manera, el transporte, "tiene una importancia capital en la sociedad actual por distintos motivos, como son permitir la movilidad, favorecer el desarrollo comercial, la competitividad y la actividad económica de las poblaciones y regiones, además de fomentar la equidad social y territorial" (Analistas Financieros Internacionales, 2012, citado en Anguita, Duarte y Flores, 2014, p. 16)

Como señala Suárez (2004, p. 70), el transporte regular de viajeros en España ocupa un papel importantísimo dentro del conjunto de diferentes modos de transporte de viajeros, el cual ha ido en un aumento derivado, entre otros factores, fundamentalmente de la modernización de infraestructuras viarias y la considerable oferta de servicios, con itinerarios que alcanzan una importante cobertura territorial.

Atendiendo a lo estipulado en el artículo 26 de la Ley 7/1985 del 2 de abril, Reguladora de las Bases del Régimen Local, los municipios con población superior a 50000 habitantes, por sí o asociados, deberán prestar, en todo caso, el servicio del transporte colectivo urbano de viajeros. Por tanto, son tales municipios los que tienen la competencia para la prestación de los servicios de transporte urbano de viajeros y pueden gestionarla de diferentes formas. Así, la referida ley, en su artículo 85, establece los diferentes modos de gestión para que los servicios públicos de competencia local puedan llevarse a cabo:

- Gestión directa, ya sea por la propia entidad local, por medio de un organismo autonómico local, una entidad pública empresarial o a través de una sociedad mercantil local cuyo capital social sea $100 \%$ de titularidad pública.

- Gestión indirecta, mediante las distintas formas previstas para el contrato de gestión de servicios públicos, en el texto refundido de la Ley de Contratos del Sector Público, aprobado por Real Decreto Legislativo 3/2011 del 14 de noviembre.

En este sentido, la Ley de Ordenación del Transporte Terrestre (LOTT), en su artículo $71,{ }^{1}$ expone:

Los transportes públicos regulares de viajeros de uso general tienen el carácter de servicios públicos de titularidad de la Administración, pudiendo ser utilizados, sin discriminación, por cualquier persona que lo desee en las condiciones establecidas en esta ley y

\footnotetext{
Este artículo se redactó según la Ley 9/2013, del 4 de julio, por la que se modifica la Ley 16/1987, del 30 de julio, de Ordenación de los Transportes Terrestres.
} 
en las normas dictadas para su ejecución y desarrollo. Como regla general, la prestación de los mencionados servicios se llevará a cabo por la empresa a la que la Administración adjudique el correspondiente contrato de gestión. No obstante, la Administración podrá optar por la gestión directa de un servicio cuando estime que resulta más adecuado al interés general en función de su naturaleza y características.

El principal problema que atañe al sector, según la literatura consultada, es la financiación. En general, la financiación del sistema de transporte público español es bastante compleja, "puesto que son varias las administraciones que intervienen y muchas las empresas operadoras de los servicios" (Delgado, Navarro, Mora y Rivero, 2013), y asimétrica, ya que "no financia por igual a las empresas operadoras de transporte de ciudades de tamaños similares" (Ruiz, 2013, p. 46). Igualmente, este último autor (2013, p. 89) señala:

A este modo desigual de financiación del transporte urbano se ha llegado con el transcurso del tiempo debido a una legislación insuficiente, y a unas administraciones públicas que no han podido resolver esta materia, ni siquiera en los años de bonanza económica, donde tal vez hubiese sido más fácil establecer principios rectores en la materia.

La financiación del transporte público urbano de viajeros se obtiene, en parte, por la recaudación directa de la venta de títulos que pagan los usuarios. Sin embargo, según afirman Martínez y Barea (2015), esta recaudación no es suficiente para cubrir los costes. Por tanto, las empresas necesitan acudir a las subvenciones que conceden las administraciones responsables del servicio. Así, tal y como comentan los referidos autores,

No solamente en España, sino en toda Europa, las Administraciones aportan importantes cantidades de dinero para cubrir la diferencia entre lo que pagan los usuarios y los costes de explotación. Y no se trata de cifras pequeñas, sino que son cantidades sustanciales en términos porcentuales y muy elevadas en términos absolutos. (p. 44)

Es por ello que, “aunque el transporte urbano es una competencia directa de los ayuntamientos, cuyas potestades legislativas están en manos de las comunidades autónomas, el Estado español se involucra de una forma significativa en la financiación del transporte" (Ruiz, 2013, p. 96).
Ser receptores de aportaciones públicas, o simplemente el hecho de prestar un servicio fundamental para el desarrollo económico y social de cualquier municipio bien justifica, a nuestro juicio, que las empresas prestatarias publicaran información, tanto sobre aspectos relevantes de la organización, como de su gestión económica y técnica. Asimismo, es obvio, como señala Lizcano (2013), que la vinculación de la sociedad actual a las redes de información es tal que mejorar la transparencia se considera un medio positivo para aumentar la confianza de los ciudadanos, ya que estos "exigen cada vez más estar suficientemente informados y tener un mayor grado de participación en las decisiones que les afectan" (p. 160)

\section{Aspectos metodológicos}

Para la consecución del objetivo de este trabajo fue necesario conformar la muestra a analizar, para lo cual se procedió a seleccionar aquellos municipios de más de 50000 habitantes en los que existen empresas prestatarias del servicio público de transporte urbano, fueran de gestión pública o privada. Así, tras la aplicación de una serie de restricciones que permitieran conformar una muestra representativa que diera respuesta a los objetivos planteados, se determinaron las empresas cuyas páginas web serían objeto de estudio.

El siguiente paso en el análisis del trabajo supuso el establecimiento de una batería de parámetros relacionados con la gestión económica, así como otros vinculados con la prestación del servicio, lo cual iba a ser objeto de estudio en las diferentes páginas web de las empresas de la muestra.

Por último, una vez establecidos los ítems a valorar, se procedió a analizar durante julio y septiembre del 2015, el contenido de las páginas web de las distintas empresas de la muestra para proceder al análisis de los resultados.

\section{La muestra}

La muestra objeto de estudio quedó conformada por empresas prestatarias del servicio en las capitales de provincias españolas, al considerarlas como las de mayor relevancia dentro de sus respectivos municipios. A partir de las 50 capitales de provincia, se estableció una primera restricción sobre el número de habitantes, a tenor de lo establecido en el referido artículo 26 de la Ley 7/1985 de 2 de abril, Reguladora de las Bases del Régimen Local, de modo que quedó en primera instancia para el 
análisis la prestación del servicio en $48^{2}$ capitales con más de 50000 habitantes.

Seguidamente, se establecieron otro tipo de restricciones con el fin de dar cumplimiento a los objetivos del trabajo, que son: 1 . El servicio de transporte debe llevarse a cabo a través de una empresa; 2 . La actividad esencial de la empresa debe ser el transporte urbano de viajeros; 3 . La empresa debe estar activa o en concurso, por lo que fue necesario utilizar también la base de datos del sABI para conocer, en ocasiones, dicha información; y 4 . Es necesario que la empresa tenga una página web, por medio de la cual se pueda examinar la información objeto de estudio.

De esta manera, la muestra queda conformada por las empresas prestatarias del servicio de transporte urbano en 40 capitales de provincia, sobre las que se procedió a realizar un análisis descriptivo de la información que proporcionan en sus páginas web.

Con el objeto de caracterizar la muestra se ha tomado de referencia, para cada una de las empresas, su antigüedad, forma jurídica, tipo de gestión y número de habitantes (tabla $1^{3}$ ). Así, se puede concluir que el $86 \%$ de las empresas se ha constituido hace más de 10 años, y que tan solo dos empresas se han constituido en los últimos cinco. Este hecho puede verse justificado, tal y como señala De Rus (2001), en que:

Las empresas privadas y públicas de transporte no han tenido que competir en el tipo de servicio que ofrecen en el mercado (transporte colectivo en autobús) al estar protegidas por barreras de entrada legales y al ser los plazos concesionales (en torno a los veinte años), suficientemente prolongados como para que la competencia potencial de nuevos aspirantes a ejercer el derecho de prestación de servicios no haya sido una amenaza real.

Con relación a la personalidad jurídica, el $70,27 \%$ de las empresas de la muestra son sociedades anónimas. Por otra parte, si se considera la forma de gestión, se puede observar que prima la gestión indirecta sobre la directa, al contar, aproximadamente, el $38 \%$ de la muestra con un capital de titularidad del $100 \%$ de la administración.

2. Se han eliminado de la muestra las capitales de Teruel y Soria por tener una cifra menor de habitantes.

3. La caracterización de la muestra se realiza exclusivamente sobre un total de 37 empresas, que son aquellas de las que se ha podido disponer de información de carácter económico.
Por último, con base en el número de habitantes de las diferentes capitales donde las empresas prestan su servicio, se ha segmentado este atendiendo a los intervalos recogidos en el INE (2014) para los diferentes tipos de estudios relacionados con la población. Además, se ha podido comprobar que dichos intervalos han sido empleados en diversos estudios, como en el realizado por la Federación Española de Municipios y Provincias (FEMP) (2009). Así, a partir de más de 50000 habitantes, resultan aplicables para nuestro trabajo tres categorías: población comprendida entre 50000 y 100000 habitantes; entre 100001 y 500000 habitantes, y, por último, superior a 500000 habitantes. De esta manera, se puede determinar que el mayor porcentaje de empresas presta servicios en municipios entre 100001 y 500000 habitantes.

Tabla 1

Características de la muestra

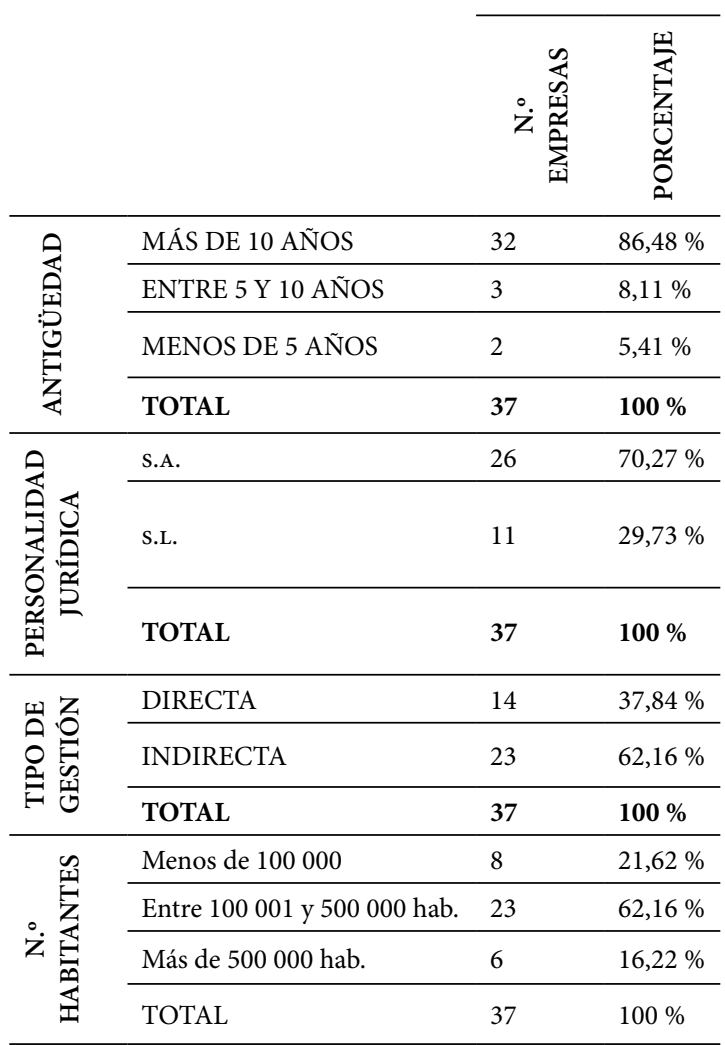

Fuente: elaboración propia. 


\section{Información objeto de análisis}

A fin de realizar un análisis descriptivo sobre la información que proporcionan las páginas web de las empresas prestatarias del servicio de transporte público urbano de pasajeros, lo primero que debemos preguntarnos es: ¿Qué se entiende por transparencia? ¿Por qué deben ser transparentes el tipo de empresas objeto de estudio? ¿Qué información económico-financiera y económico-técnica deberían presentar para conseguir ser transparentes a través de sus páginas web?

Como señalan Alonso y García (2014), no existe "una definición única de transparencia en la literatura, todas las aproximaciones que se han hecho a ella giran en torno al concepto básico de suministrar un amplio caudal de información relevante al público en plazos oportunos de tiempo" (p. 76). Así, autores como Del Campo, Herrador y Segovia, (2015, p. 65) señalan:

La transparencia es un concepto relacionado con la posibilidad de que la información de una empresa, gobierno u organización, pueda ser consultada por los diferentes agentes interesados, de tal modo que éstos puedan tomar decisiones sin estar sometidos a una asimetría informativa.

Desde un prisma legal, la Ley 19/2013, de 9 de diciembre, de transparencia, acceso a la información pública y buen gobierno, en su artículo 2 recoge que el ámbito de aplicación de la misma es de aplicación a la administración local. De igual modo, en el citado artículo también se recoge que les será de aplicación esta ley a "las sociedades mercantiles en cuyo capital social la participación, directa o indirecta, de las entidades previstas en este artículo sea superior al 50 por 100 ". Surge, como se puede deducir, un problema de asimetría en la demanda de transparencia de las empresas prestatarias del servicio de transporte público de viajeros. Mientras que las sociedades mercantiles con participación superior al 50 \% están obligadas a la norma, el resto no.

Por otra parte, la mencionada Ley 19/2013, recoge en su artículo 5 que la "información sujeta a las obligaciones de transparencia será publicada en las correspondientes sedes electrónicas o páginas web y de una manera clara, estructurada y entendible para los interesados". Por tanto, se entiende que las páginas web son la herramienta que sustenta las obligaciones de transparencia recogidas en la ley.
En suma, no cabe duda de que la administración pública del servicio y el derecho legal de los ciudadanos a su prestación exige que los distintos grupos de interés puedan conocer no solo información comercial para acceder al servicio, sino también de tipo técnico o económico, en función de sus intereses. Son, por tanto, motivos más que suficientes para exigir transparencia a las empresas del sector el hecho de ser prestatarias de un servicio público y receptoras de recursos públicos.

Tras realizar una búsqueda bibliográfica sobre literatura en el ámbito de la transparencia sobre el sector del transporte urbano regular de pasajeros en España, encontramos el trabajo proporcionado por Escamilla, Plaza y Flores (2015). Estas autoras han centrado su estudio en observar si las empresas de transporte público urbano de titularidad pública, es decir, gestionadas de forma directa, divulgan información sobre responsabilidad social empresarial. Para ello, analizan las páginas web de 17 empresas de este sector en España, ya que,

La sociedad exige que las actuaciones de las entidades públicas sean sostenibles, eficiente y transparentes en su gestión. (...) [Dichas exigencias] han derivado, tanto en la aprobación, a nivel nacional, de la Ley de Transparencia como en iniciativas, a nivel europeo, que abogan por la importancia de la difusión de información de RsC en dichas entidades. (Escamilla et al., 2015, p. 196)

Así, en resumidas cuentas, se puede establecer, bajo la investigación realizada, que las empresas ofrecen escasa información sobre responsabilidad social empresarial en sus páginas web, dado que tan solo el 23,53\% de las empresas contemplan de 14 a 27 ítems analizados.

En nuestro trabajo, adoptamos como punto de partida para establecer las variables o ítems a analizar, los indicadores contenidos en el informe del Observatorio de Costes y Financiación del Transporte Urbano Colectivo (отUC) (2015), ya que, como se afirma en la carta de presentación, los mismos permiten proporcionar:

Al gestor la posibilidad de llevar a cabo estudios económico-financieros sobre la situación de su empresa respecto al resto del sector (...) y, por tanto, muy útiles para obtener conclusiones de comportamientos de las variables, evolución, etc., fundamental para estudios 
de carácter más estratégico y las correspondientes reflexiones de los responsables políticos de las mismas. Con ello, se seleccionaron los diferentes ítems (un total de 30) a utilizar para analizar la información contenida en las páginas web de las empresas (tabla 2). Asimismo, se procedió en línea con la literatura precedente (Delgado, Navarro, Mora y Rivero, 2014; Haro et al., 2013; Transparencia Internacional España, 2014), a organizarlos de forma que sirviera de base para realizar la descripción de los contenidos en las páginas web, a efectos, en nuestro caso, de analizar la información divulgada. Asimismo, se procedió a su clasificación en las dimensiones: actividad, medios de producción técnicos, consumos, medios humanos e información económica. Junto con tales dimensiones se consideró oportuno añadir otra referida a la organización de las empresas, a efecto de proporcionar otro tipo de conclusiones basadas en sus características organizativas. En la tabla 2 se muestran los 39 ítems a evaluar, organizados en las seis dimensiones y codificados utilizando la inicial de la correspondiente denominación y un número correlativo por dimensión.

El análisis del contenido de cada una de las páginas web de las empresas de la muestra se ha llevado a cabo a través de la asignación a cada ítem de una puntuación de 1 o 0 puntos. Se asigna el 1 cuando la información se recoge en la página web, y un 0 en caso contrario.

En cuanto a la recopilación y tratamiento de la información, se optó por aplicar a cada web el cuestionario de ítems por parte de un primer observador, y se registró el resultado en una hoja de cálculo, en la que se presenta como cuadro de doble entrada - capital de provincia y código de cada ítem-. Luego, se procedió a su comprobación por parte del resto de observadores, adoptando, en caso de diferencias, las oportunas decisiones en torno a la fijación del valor a considerar en cada celda. Por último, se determina el número de empresas que divulga cada ítem analizado.

Tabla 2

Ítems a analizar agrupados en dimensiones

\begin{tabular}{|c|c|c|}
\hline DIMENSIÓN & & ÍTEMS \\
\hline \multirow{9}{*}{ DIMENSIÓN 1: ORGANIZACIÓN } & $\mathrm{O} 1$ & Misión, visión y valores \\
\hline & $\mathrm{O} 2$ & Organigrama de la empresa \\
\hline & $\mathrm{O} 3$ & Composición del Consejo de Administración \\
\hline & $\mathrm{O} 4$ & cv de los miembros del Consejo de Administración \\
\hline & O5 & Identificación del directivo de más alto nivel \\
\hline & O6 & Identificación del equipo directivo de la empresa \\
\hline & O7 & Número de trabajadores de la compañía \\
\hline & $\mathrm{O} 8$ & Forma jurídica \\
\hline & O9 & Tipo de gestión \\
\hline \multirow{7}{*}{ DIMENSIÓN 2: ACTIVIDAD } & A1 & Viajeros transportados \\
\hline & A2 & Kilómetros recorridos \\
\hline & A3 & Km de carril bus \\
\hline & A4 & Número de líneas \\
\hline & A5 & Puntos de paradas totales \\
\hline & A6 & Velocidad red \\
\hline & A7 & Número de viajes/semiviajes \\
\hline \multirow{5}{*}{$\begin{array}{l}\text { DIMENSIÓN 3: MEDIOS DE } \\
\text { PRODUCCIÓN TÉCNICOS }\end{array}$} & MT1 & Número de autobuses \\
\hline & MT2 & Antigüedad de la flota \\
\hline & MT3 & Número de plazas ofertadas \\
\hline & MT4 & Autobuses por tipo de combustible \\
\hline & MT5 & Tipología de autobuses \\
\hline \multirow{2}{*}{ DIMENSIÓN 4: CONSUMOS } & $\mathrm{C} 1$ & Consumo combustible \\
\hline & $\mathrm{C} 2$ & Consumo energético \\
\hline
\end{tabular}




\begin{tabular}{|c|c|c|}
\hline DIMENSIÓN & & ÍTEMS \\
\hline \multirow{3}{*}{ DIMENSIÓN 5: MEDIOS HUMANOS } & MH1 & Número de conductores \\
\hline & $\mathrm{MH} 2$ & Información diferenciada de otro tipo de personal \\
\hline & $\mathrm{MH} 3$ & Absentismo \\
\hline \multirow{13}{*}{ DIMENSIÓN 6: INFORMACIÓN ECONÓMICA } & E1 & Balance \\
\hline & E2 & Cuenta Pyg \\
\hline & E3 & efe \\
\hline & E4 & Informe de gestión \\
\hline & E5 & Memoria \\
\hline & E6 & Recaudación directa del viajero \\
\hline & E7 & Montante de aportaciones públicas recibidas \\
\hline & E8 & Tipología de la subvención/aportación recibida \\
\hline & E9 & Coeficiente de endeudamiento \\
\hline & E10 & Informe de auditoría \\
\hline & E11 & Presupuesto de la entidad \\
\hline & E12 & Coste promedio por conductor \\
\hline & E13 & Tarifa \\
\hline
\end{tabular}

Fuente: elaboración propia.

\section{Resultados del estudio}

El estudio se centra en describir la información a través de cada una de las seis dimensiones anteriormente comentadas, de forma que proporcione las bases y parámetros de comparación, con el fin de dar respuesta al objetivo primordial del estudio y obtener las conclusiones principales que de este se deriven. Por otro lado, una vez completado el primer análisis realizado para el conjunto de las empresas, también se ha incluido un análisis segmentado en función del tipo de gestión, así como del número de habitantes, criterios de los que se pudo obtener información para la totalidad de las empresas.

\section{Resultados procedentes del análisis a nivel global}

Tras el análisis de las 40 páginas web, se observa cómo no todas las empresas reconocen prestar, fundamentalmente, el servicio de transporte urbano en las correspondientes capitales de provincia, por lo que fue necesario acudir para obtener esta información del resto de empresas a otras fuentes distintas a su web. Los resultados obtenidos tras analizar los diferentes ítems en las páginas web son los que se muestran en la tabla 3. Como primer resultado, se puede destacar que no existe ninguna empresa que aporte información sobre todos los ítems (39); tan solo la empresa prestataria del servicio en Palma de Mallorca consigue mostrar 33 en su web.

Se puede observar cómo a nivel de dimensiones, con carácter general, las empresas aportan más información de ítems clasificados en las de organización y actividad. En un análisis por ítem, las tarifas y el número de líneas constituyen los únicos sobre los que la totalidad de las empresas proporcionan información. Esto se puede explicar por el contenido de carácter comercial de muchas de las páginas web, a efectos de que incluso en alguna de ellas se permita adquirir los títulos por este medio. Además, cabe destacar que el número de paradas totales es el siguiente ítem sobre el que mayor información facilitan las empresas, si bien en la mayor parte de las mismas esta información no se expresa directamente en la web, sino que se puede obtener por agregación de las paradas de cada línea. Con un sentido opuesto, se observa que ninguna empresa aporta información sobre el coste promedio por conductor.

Al centrarse en cada una de las dimensiones, se destaca, a nivel de la organización, cómo ninguna empresa aporta la totalidad de los ítems que delimitan esta dimensión. También se aprecia cómo se encuentran empresas que en sus páginas web no incluyen 
ningún ítem de esta dimensión (15\% de la muestra). Además, los referidos a la forma jurídica y al tipo de gestión, son los de mayor presencia en las páginas web $y$, por el contrario, el cv de los miembros del consejo de administración es el de menor presencia, pues tan solo una empresa lo aporta.

Con respecto a la dimensión sobre la actividad se observa cómo tampoco hay empresas que aporten información sobre la totalidad de los ítems analizados. Como se ha comentado, son el número de las líneas que ofertan y los puntos de paradas totales sobre los que mayor número de empresas facilitan información y, en contraposición, encontramos el número de viajes/ semiviajes, variables sobre las que muy pocas empresas divulgan información.

Por otra parte, de los medios de producción técnicos, en torno al $48 \%$ de las empresas no proporciona información alguna de esta dimensión. Asimismo, se observa cómo para esta dimensión cuatro empresas que aportan toda la información de la dimensión. El número de autobuses encabeza el ranquin de presencia, pero simplemente se aporta el número total sin discriminar el número de plazas potenciales que pueden ofertar, pues tan solo cuatro empresas informan de ello.
Del análisis de la dimensión sobre consumos es mucho mayor el porcentaje de empresas que no divulga información sobre los consumos de combustible y energía (87,5 \% de la muestra). Es, también, como ocurría en la dimensión anterior, relativamente bajo el número de empresas que presenta información sobre ambos ítems, tan solo cuatro (10 \% de la muestra).

Con respecto a la dimensión sobre medios humanos, tan solo tres empresas muestran información de todos los ítems analizados, y 31 es el número de empresas que no publica ningún ítem. Las variables sobre las que se aporta mayor información son el número de conductores y el tipo de personal, pero son muy pocas empresas las que aportan el dato.

Finalmente, si atendemos a la dimensión sobre información económica, las tarifas y el coste promedio por conductor, tal y como se comentó anteriormente, son, respectivamente, aquellos ítems sobre los que se aporta más - $100 \%$ de la muestra-, y menos información (ninguna empresa de la muestra). Se destaca cómo son escasas las empresas que publican sus cuentas anuales en la web, e incluso las que aportan el dato sobre las aportaciones públicas recibidas para la prestación del servicio.

Tabla 3

Resultados obtenidos de las dimensiones a nivel global

\begin{tabular}{|c|c|c|c|c|}
\hline DIMENSIÓN & & ÍTEMS & EMPRESAS & $\%$ \\
\hline \multirow{9}{*}{ DIMENSIÓN 1: ORGANIZACIÓN } & $\mathrm{O}_{1}$ & Misión, visión y valores & 10 & $25,00 \%$ \\
\hline & $\mathrm{O}_{2}$ & Organigrama de la empresa & 8 & $20,00 \%$ \\
\hline & $\mathrm{O}_{3}$ & Composición del consejo de administración & 11 & $27,00 \%$ \\
\hline & $\mathrm{O}_{4}$ & Cv de los miembros del consejo de administración & 1 & $2,50 \%$ \\
\hline & $\mathrm{O}_{5}$ & Identificación del directivo de más alto nivel & 8 & $20,00 \%$ \\
\hline & O6 & Identificación del equipo directivo de la empresa & 7 & $17,50 \%$ \\
\hline & $\mathrm{O}_{7}$ & Número de trabajadores de la compañía & 13 & $32,50 \%$ \\
\hline & O8 & Forma jurídica & 26 & $65,00 \%$ \\
\hline & $\mathrm{O}_{9}$ & Tipo de gestión & 25 & $62,50 \%$ \\
\hline \multirow{7}{*}{ DIMENSIÓN 2: ACTIVIDAD } & A1 & Viajeros transportados & 15 & $37,50 \%$ \\
\hline & A2 & Kilómetros recorridos & 12 & $30,00 \%$ \\
\hline & $\mathrm{A}_{3}$ & Km de carril bus & 5 & $12,50 \%$ \\
\hline & $\mathrm{A}_{4}$ & Número de líneas & 37 & $92,50 \%$ \\
\hline & $\mathrm{A}_{5}$ & Puntos de paradas totales & 35 & $87,50 \%$ \\
\hline & A6 & Velocidad red & 7 & $17,50 \%$ \\
\hline & A7 & Número de viajes/semiviajes & 2 & $5,00 \%$ \\
\hline
\end{tabular}




\begin{tabular}{|c|c|c|c|c|}
\hline DIMENSIÓN & & ÍTEMS & EMPRESAS & $\%$ \\
\hline \multirow{5}{*}{$\begin{array}{l}\text { DIMENSIÓN 3: MEDIOS DE } \\
\text { PRODUCCIÓN TÉCNICOS }\end{array}$} & $\mathrm{MT}_{1}$ & Número de autobuses & 21 & $52,50 \%$ \\
\hline & $\mathrm{MT}_{2}$ & Antigüedad de la flota & 10 & $25,00 \%$ \\
\hline & $\mathrm{MT}_{3}$ & Número de plazas ofertadas & 4 & $10,00 \%$ \\
\hline & $\mathrm{MT}_{4}$ & Autobuses por tipo de combustibles & 10 & $25,00 \%$ \\
\hline & $\mathrm{MT}_{5}$ & Tipología de autobuses & 10 & $25,00 \%$ \\
\hline \multirow{2}{*}{ DIMENSIÓN 4: CONSUMOS } & $\mathrm{C}_{1}$ & Consumo combustible & 5 & $25,50 \%$ \\
\hline & $\mathrm{C}_{2}$ & Consumo energético & 4 & $10,00 \%$ \\
\hline \multirow{3}{*}{$\begin{array}{l}\text { DIMENSIÓN 5: MEDIOS } \\
\text { HUMANOS }\end{array}$} & $\mathrm{MH} 1$ & Número de conductores & 8 & $20,00 \%$ \\
\hline & $\mathrm{MH}_{2}$ & Información diferenciada de otro tipo de personal & 9 & $22,50 \%$ \\
\hline & $\mathrm{MH}_{3}$ & Absentismo & 3 & $7,50 \%$ \\
\hline \multirow{13}{*}{$\begin{array}{l}\text { DIMENSIÓN 6: INFORMACIÓN } \\
\text { ECONÓMICA }\end{array}$} & E1 & Balance & 8 & $20,00 \%$ \\
\hline & E2 & Cuenta de P y G & 8 & $20,00 \%$ \\
\hline & $\mathrm{E}_{3}$ & EFE & 5 & $12,50 \%$ \\
\hline & $\mathrm{E}_{4}$ & Informe de gestión & 5 & $12,50 \%$ \\
\hline & $\mathrm{E}_{5}$ & Memoria & 4 & $10,00 \%$ \\
\hline & E6 & Recaudación directa del viajero & 3 & $7,50 \%$ \\
\hline & E7 & Montante de aportaciones públicas recibidas & 5 & $12,50 \%$ \\
\hline & E8 & Tipología de la subvención/aportación recibida & 2 & $5,00 \%$ \\
\hline & E9 & Coeficiente de endeudamiento & 1 & $2,50 \%$ \\
\hline & E10 & Informe de auditoría & 4 & $10,00 \%$ \\
\hline & E11 & Presupuesto de la entidad & 3 & $7,50 \%$ \\
\hline & E12 & Coste promedio por conductor & 0 & $0,00 \%$ \\
\hline & E13 & Tarifa & 37 & $92,50 \%$ \\
\hline
\end{tabular}

Fuente: elaboración propia.

\section{Resultados obtenidos de la segmentación de la muestra}

En este apartado se exponen los resultados del descriptivo tras segmentar la muestra por el tipo de gestión y el tamaño. El tipo de gestión es un criterio relevante en la prestación de un servicio público. Además, en la Ley de Transparencia se otorgan implicaciones informativas distintas para las sociedades mercantiles cuyo capital es $100 \%$ de titularidad pública, y para aquellos otros operadores que gestionan el transporte urbano con capital privado y reciben aportaciones públicas. Este criterio es uno de los analizados por la FEMP (2009), en su informe publicado para tratar de identificar los factores determinantes de la prestación del servicio de transporte público urbano en España.
Otro criterio que también se utiliza en el referido informe de la FEMP (2009) es el del tamaño poblacional, ya que tras el estudio llevado a cabo, esta variable se encuentra fuertemente correlacionada con otras 14 , entre las que se puede destacar el número de viajeros trasportados, el número de kilómetros recorridos, el número de kilómetros de la red, el número de plazas ofrecidas, el número de empleados, el número de paradas y el número de autobuses. Por otra parte, de acuerdo con Cárcaba y García (2014, p. 72):

Los ayuntamientos más grandes no sólo son los que cuentan con un mayor número de habitantes, sino también los que ocupan un territorio más amplio, por lo que se verán alentados a acudir a la Red para hacer llegar la información financiera a los ciudadanos. 
En cuanto a la segmentación de la muestra, de acuerdo con la forma en la que se gestiona el servicio, de las 40 empresas, 26 son las que prestan el servicio con capital privado (gestión indirecta), y 14 con el $100 \%$ de titularidad pública (gestión directa). Por otra parte, en cuanto al tamaño poblacional, 10 son las empresas de capitales de menor dimensión (menos de 100000 habitantes), 24 en el intervalo de 100001 a 500000 habitantes, y seis en las de mayor dimensión (más de 500000 habitantes).

Tabla 4

Empresas según tipo de gestión y número de habitantes
Cárcaba y García (2014) afirman cómo, “cuanto más población tenga una ciudad, más probable es que la empresa que preste el servicio de transporte urbano sea pública". A este respecto, podemos observar en la tabla 4, de qué manera en las capitales de mayor dimensión el servicio los prestan, principalmente, empresas públicas, y en las de menor dimensión se gestiona más de forma indirecta.

\begin{tabular}{cccc}
\hline & $<100000$ & entre 100001 y 500000 & $>500000$ \\
\hline DIRECTA & 1 & 8 & 5 \\
\hline INDIRECTA & 9 & 16 & 1 \\
\hline
\end{tabular}

Fuente: elaboración propia.

Los resultados obtenidos de la segmentación de la muestra de acuerdo con la forma de gestión y el número de habitantes de las diferentes capitales, se sintetizan de la tablas 5 y 6 . En estas se ha obviado el coste promedio por conductor, ya que, como se señaló anteriormente, ninguna empresa comunica este tipo de información. Tampoco se han incluido los ítems de las tarifas y el número de líneas, puesto que, por el contrario, los proporcionan la totalidad de las empresas.

En la tabla 5 se muestra el porcentaje de empresas de gestión directa e indirecta que divulga información de cada uno de los ítems analizados. Tales porcentajes ponen de relieve que, con carácter general, las empresas que se gestionan directamente por medio de la administración competente, presentan mayor información que aquellas gestionadas de forma indirecta, lo cual pone de manifiesto que, tal y como hemos comentado, en ambos casos no existe ninguna empresa que proporcione información de la totalidad de los ítems. Asimismo, cabe resaltar cómo ninguna empresa que presta el servicio con capital privado publica en su web información sobre aspectos económicos.

Tabla 5

Resultados obtenidos a través de la segmentación por tipo de gestión

DIMENSIÓN 1: ORGANIZACIÓN

\begin{tabular}{|c|c|c|c|c|c|}
\hline & & \multirow{2}{*}{\multicolumn{4}{|c|}{ GESTIÓN }} \\
\hline & & & & & \\
\hline & & \multicolumn{2}{|l|}{ DIRECTA } & \multicolumn{2}{|l|}{ INDIRECTA } \\
\hline & & EMPRESAS & $\%$ & EMPRESAS & $\%$ \\
\hline $\mathrm{O} 1$ & Misión, visión y valores & 7 & $50 \%$ & 3 & $12 \%$ \\
\hline $\mathrm{O} 2$ & Organigrama de la empresa & 7 & $50 \%$ & 1 & $4 \%$ \\
\hline O3 & $\begin{array}{l}\text { Composición del consejo de } \\
\text { administración }\end{array}$ & 11 & $79 \%$ & 0 & $0 \%$ \\
\hline $\mathrm{O} 4$ & $\begin{array}{l}\text { CV de los miembros del consejo de } \\
\text { Administración }\end{array}$ & 1 & $7 \%$ & 0 & $0 \%$ \\
\hline O5 & $\begin{array}{l}\text { Identificación del directivo de más } \\
\text { alto nivel }\end{array}$ & 8 & $57 \%$ & 0 & $0 \%$ \\
\hline O6 & $\begin{array}{l}\text { Identificación del equipo directivo de } \\
\text { la empresa }\end{array}$ & 7 & $50 \%$ & 0 & $0 \%$ \\
\hline O7 & $\begin{array}{l}\text { Número de trabajadores de la } \\
\text { compañía }\end{array}$ & 10 & $71 \%$ & 3 & $12 \%$ \\
\hline O8 & Forma jurídica & 12 & $86 \%$ & 15 & $58 \%$ \\
\hline O9 & Tipo de gestión & 11 & $79 \%$ & 16 & $62 \%$ \\
\hline
\end{tabular}




\begin{tabular}{|c|c|c|c|c|c|c|}
\hline & & & \multicolumn{4}{|l|}{ GESTIÓN } \\
\hline & & & \multicolumn{2}{|l|}{ DIRECTA } & \multicolumn{2}{|l|}{ INDIRECTA } \\
\hline & & & EMPRESAS & $\%$ & EMPRESAS & $\%$ \\
\hline \multirow{6}{*}{ DIMENSIÓN 2: ACTIVIDAD } & A1 & Viajeros transportados & 8 & $57 \%$ & 7 & $27 \%$ \\
\hline & $\mathrm{A} 2$ & Kilómetros recorridos & 8 & $57 \%$ & 4 & $15 \%$ \\
\hline & A3 & Km de carril bus & 5 & $36 \%$ & 0 & $0 \%$ \\
\hline & A5 & Puntos de paradas totales & 14 & $100 \%$ & 26 & $100 \%$ \\
\hline & A6 & Velocidad red & 6 & $43 \%$ & 24 & $92 \%$ \\
\hline & A7 & Número de viajes/semiviajes & 2 & $14 \%$ & 1 & $4 \%$ \\
\hline \multirow{5}{*}{$\begin{array}{l}\text { DIMENSIÓN3: } \\
\text { MEDIOS DE PRODUCCIÓN } \\
\text { TÉCNICOS }\end{array}$} & MT1 & Número de autobuses & 11 & $79 \%$ & 10 & $38 \%$ \\
\hline & MT2 & Antigüedad de la flota & 7 & $50 \%$ & 3 & $12 \%$ \\
\hline & MT3 & Número de plazas ofertadas & 4 & $29 \%$ & 0 & $0 \%$ \\
\hline & MT4 & Autobuses por tipo de combustibles & 9 & $64 \%$ & 1 & $4 \%$ \\
\hline & MT5 & Tipología de autobuses & 8 & $57 \%$ & 2 & $8 \%$ \\
\hline \multirow{2}{*}{ DIMENSIÓN 4: CONSUMOS } & $\mathrm{C} 1$ & Consumo combustible & 5 & $36 \%$ & 0 & $0 \%$ \\
\hline & $\mathrm{C} 2$ & Consumo energético & 4 & $29 \%$ & 0 & $0 \%$ \\
\hline \multirow{3}{*}{$\begin{array}{l}\text { DIMENSIÓN 5: } \\
\text { MEDIOS HUMANOS }\end{array}$} & MH1 & Número de conductores & 8 & $57 \%$ & 0 & $0 \%$ \\
\hline & $\mathrm{MH} 2$ & $\begin{array}{l}\text { Información diferenciada de otro tipo } \\
\text { de personal }\end{array}$ & 9 & $64 \%$ & 0 & $0 \%$ \\
\hline & $\mathrm{MH} 3$ & Absentismo & 3 & $21 \%$ & 0 & $0 \%$ \\
\hline \multirow{11}{*}{$\begin{array}{l}\text { DIMENSIÓN 6: } \\
\text { INFORMACIÓN } \\
\text { ECONÓMICA }\end{array}$} & $\mathrm{E} 1$ & Balance & 8 & $57 \%$ & 0 & $0 \%$ \\
\hline & E2 & Cuenta de Pyg & 8 & $57 \%$ & 0 & $0 \%$ \\
\hline & E3 & EFE & 5 & $36 \%$ & 0 & $0 \%$ \\
\hline & $\mathrm{E} 4$ & Informe de gestión & 5 & $36 \%$ & 0 & $0 \%$ \\
\hline & E5 & Memoria & 4 & $29 \%$ & 0 & $0 \%$ \\
\hline & E6 & Recaudación directa del viajero & 3 & $21 \%$ & 0 & $0 \%$ \\
\hline & E7 & $\begin{array}{l}\text { Montante de aportaciones públicas } \\
\text { recibidas }\end{array}$ & 5 & $36 \%$ & 0 & $0 \%$ \\
\hline & E8 & $\begin{array}{l}\text { Tipología de la subvención/aportación } \\
\text { recibida }\end{array}$ & 2 & $14 \%$ & 0 & $0 \%$ \\
\hline & E9 & Coeficiente de endeudamiento & 1 & $7 \%$ & 0 & $0 \%$ \\
\hline & E10 & Informe de auditoría & 4 & $29 \%$ & 0 & $0 \%$ \\
\hline & E11 & Presupuesto de la entidad & 3 & $21 \%$ & 0 & $0 \%$ \\
\hline
\end{tabular}

Fuente: elaboración propia.

Con respecto a la relación entre el tamaño de la población y la información contable en Internet, Cárcaba y García (2008) contrastan en su trabajo cómo cuanto mayor es el tamaño de los municipios, mayor es la necesidad de divulgar información financiera, ya que existe una relación positiva entre ambos. Como se muestra en la tabla 6, las empresas que prestan su servicio en capitales de menos de 100000 habitantes divulgan menos información en sus páginas web con relación a aquellas otras dos categorías de segmentación. No obstante, dentro de las primeras destaca una compañía por el número de ítems que publica en su web - un total de 12-, patrón al que no responde el resto de empresas de este segmento, las cuales divulgan como máximo cinco de los ítems analizados. Asimismo, la empresa que publica mayor número de ítems pertenece al tamaño intermedio. 
Tabla 6

Resultados obtenidos a través de la segmentación por número de habitantes

\begin{tabular}{|c|c|c|c|c|c|c|c|}
\hline & \multicolumn{7}{|c|}{ N. ${ }^{\circ}$ HABITANTES } \\
\hline & $<100000$ hab. & & Entre 10000 & 500000 hab. & $>500000$ hal & & \\
\hline & EMPRESAS & $\%$ & EMPRESAS & $\%$ & EMPRESAS & $\%$ & \\
\hline $\mathrm{O} 1$ & Misión, visión y valores & 0 & $0 \%$ & 5 & $21 \%$ & 5 & $83 \%$ \\
\hline $\mathrm{O} 2$ & Organigrama de la empresa & 0 & $0 \%$ & 4 & $17 \%$ & 4 & $67 \%$ \\
\hline $\mathrm{O} 3$ & Composición del consejo de administración & 0 & $0 \%$ & 6 & $25 \%$ & 5 & $83 \%$ \\
\hline $\mathrm{O} 4$ & Cv de los miembros del consejo de administración & 0 & $0 \%$ & 0 & $0 \%$ & 1 & $17 \%$ \\
\hline O5 & Identificación del directivo de más alto nivel & 0 & $0 \%$ & 4 & $17 \%$ & 4 & $67 \%$ \\
\hline O6 & Identificación del equipo directivo de la empresa & 0 & $0 \%$ & 4 & $17 \%$ & 3 & $50 \%$ \\
\hline O7 & Número de trabajadores de la compañía & 0 & $0 \%$ & 8 & $33 \%$ & 5 & $83 \%$ \\
\hline O8 & Forma jurídica & 5 & $0 \%$ & 16 & $67 \%$ & 6 & $100 \%$ \\
\hline O9 & Tipo de gestión & 4 & $40 \%$ & 17 & $71 \%$ & 6 & $100 \%$ \\
\hline A1 & Viajeros transportados & 0 & $0 \%$ & 10 & $42 \%$ & 5 & $83 \%$ \\
\hline A2 & Kilómetros recorridos & 0 & $0 \%$ & 8 & $33 \mathrm{u} \%$ & 4 & $67 \%$ \\
\hline $\mathrm{A} 3$ & Km de carril bus & 0 & $0 \%$ & 1 & $4 \%$ & 4 & $67 \%$ \\
\hline A5 & Puntos de paradas totales & 10 & $100 \%$ & 22 & $92 \%$ & 6 & $100 \%$ \\
\hline A6 & Velocidad red & 0 & $0 \%$ & 3 & $13 \%$ & 4 & $67 \%$ \\
\hline A7 & Número de viajes/semiviajes & 0 & $0 \%$ & 2 & $8 \%$ & 0 & $0 \%$ \\
\hline MT1 & Número de autobuses & 1 & $10 \%$ & 14 & $58 \%$ & 6 & $100 \%$ \\
\hline MT2 & Antigüedad de la flota & 0 & $0 \%$ & 6 & $25 \%$ & 4 & $67 \%$ \\
\hline MT3 & Número de plazas ofertadas & 0 & $0 \%$ & 3 & $13 \%$ & 1 & $17 \%$ \\
\hline MT4 & Autobuses por tipo de combustibles & 0 & $0 \%$ & 5 & $21 \%$ & 5 & $83 \%$ \\
\hline MT5 & Tipología de autobuses & 1 & $10 \%$ & 4 & $17 \%$ & 5 & $83 \%$ \\
\hline $\mathrm{C} 1$ & Consumo combustible & 0 & $0 \%$ & 3 & $13 \%$ & 2 & $33 \%$ \\
\hline $\mathrm{C} 2$ & Consumo energético & 0 & $0 \%$ & 2 & $8 \%$ & 2 & $33 \%$ \\
\hline MH1 & Número de conductores & 0 & $0 \%$ & 4 & $17 \%$ & 4 & $67 \%$ \\
\hline MH2 & Información diferenciada de otro tipo de personal & 1 & $10 \%$ & 4 & $17 \%$ & 4 & $67 \%$ \\
\hline MH3 & Absentismo & 0 & $0 \%$ & 2 & $8 \%$ & 1 & $17 \%$ \\
\hline E1 & Balance & 1 & $10 \%$ & 3 & $13 \%$ & 4 & $67 \%$ \\
\hline E2 & Cuenta de Pyg & 1 & $10 \%$ & 3 & $13 \%$ & 4 & $67 \%$ \\
\hline E3 & EFE & 1 & $10 \%$ & 2 & $8 \%$ & 2 & $33 \%$ \\
\hline $\mathrm{E} 4$ & Informe de gestión & 0 & $0 \%$ & 3 & $13 \%$ & 2 & $33 \%$ \\
\hline E5 & Memoria & 1 & $10 \%$ & 1 & $4 \%$ & 2 & $33 \%$ \\
\hline E6 & Recaudación directa del viajero & 0 & $0 \%$ & 1 & $4 \%$ & 2 & $33 \%$ \\
\hline E7 & Montante de aportaciones públicas recibidas & 1 & $10 \%$ & 2 & $8 \%$ & 2 & $33 \%$ \\
\hline E8 & Tipología de la subvención/aportación recibida & 0 & $0 \%$ & 1 & $4 \%$ & 1 & $17 \%$ \\
\hline E9 & Coeficiente de endeudamiento & 0 & $0 \%$ & 0 & $0 \%$ & 1 & $17 \%$ \\
\hline E10 & Informe de auditoría & 1 & $10 \%$ & 2 & $8 \%$ & 1 & $17 \%$ \\
\hline E11 & Presupuesto de la entidad & 0 & $0 \%$ & 2 & $8 \%$ & 1 & $17 \%$ \\
\hline
\end{tabular}

Fuente: elaboración propia. 


\section{Conclusiones}

El transporte público urbano colectivo de viajeros es hoy en día una necesidad básica para el bienestar de los ciudadanos y la economía del país. Es por esto que aquellos municipios de más de 50000 habitantes se encuentran obligados a prestar el servicio del transporte, como forma de cubrir las necesidades de movilidad de la sociedad.

En las capitales de provincias españolas este servicio se presta, fundamentalmente, a través de empresas con una antigüedad superior a 10 años y con una mayor presencia de gestión indirecta.

Con el análisis realizado se ha pretendido cubrir el objetivo de describir la forma de organizar del transporte urbano en las capitales de provincias españolas, así como la información, tanto económica, como técnica, contenida en las páginas web de las empresas prestatarias del servicio de transporte de viajeros. Todo esto sin entrar a valorar su accesibilidad ni calidad. Cabe señalar que no hay ninguna empresa que proporcione la totalidad de ítems a evaluar, ya que la empresa que más información aporta consigue recoger en su páginas web un total de 33 ítems.

Los resultados obtenidos ponen de manifiesto la escasa información económica que aportan las empresas en sus páginas web, y se destacan las aportaciones públicas recibidas, ya que tan solo cinco empresas publican tal información. Esta variable es clave para estar en capacidad de evaluar la gestión realizada, ya que, como se señala en отUc (2015), el que una empresa tenga un mayor resultado, no significa que esté realizando mejor su gestión, debido a que habría que tener en cuenta la forma en que cada una recibe las aportaciones por parte de la administración. Está claro que ello afectará a la rentabilidad de estas empresas.

Asimismo, dada la heterogeneidad innata en el sector, resulta fundamental, a fin de evaluar la gestión y realizar benchmarking entre empresas, dar a conocer determinadas variables que permitan relativizar el concepto de gasto económico incurrido en la prestación del servicio. A este respecto, existen gastos que pueden estar vinculados con el número de viajeros o el número de kilómetros y, sin embargo, de tales indicadores la mayoría de las empresas no proporciona información en sus páginas web; tan solo un $30 \%$ de la muestra aporta información sobre los kilómetros recorridos y, en torno a un $40 \%$ de los viajeros transportados.
Por el contrario, de los diferentes ítems analizados, los resultados nos llevan a señalar que las tarifas y el número de líneas son las variables sobre las que todas las empresas proporcionan información, ya sea con mayor o menor accesibilidad a esta. Este alto grado de aportación por parte de las empresas sobre dicha información puede verse justificada en el carácter comercial de la web. En general, podemos destacar que solo existen seis ítems sobre los que más de la mitad de las empresas proporciona información, los cuales son, junto con los dos anteriores, la forma jurídica, el tipo de gestión, los puntos de paradas totales y el número de autobuses. Además, en contraposición a estos ítems, encontramos el coste promedio por conductor del que no se ha encontrado presencia en ninguna web.

Si atendemos a los resultados fruto de la segmentación, se llega a la conclusión de que las empresas gestionadas de forma directa proporcionan más información que aquellas gestionadas de forma indirecta. Esto se evidencia en que el promedio de ítems publicado en las empresas de gestión directa es de 19, mientras que en las de gestión indirecta es tan solo de 5.

Por otro lado, en cuanto a la segmentación relacionada con el número de habitantes de las diferentes capitales, las empresas que prestan su servicio en poblaciones inferiores a 100000 habitantes son las que proporcionan menor información sobre los diferentes ítems, con un promedio de 5 ítems, mientras que en las de mayor población el promedio alcanza los 22 ítems.

Con todo ello, se puede observar cómo la información que publican en las páginas web las empresas prestatarias del servicio es muy escasa, $y$, en ocasiones, poco consistente. Así, el presente trabajo contribuye a la literatura sobre transparencia de las empresas de transporte urbano de viajeros, al ampliar el campo de actuación del trabajo publicado por Escamilla et al. (2015), tanto en cuanto al número de empresas analizadas, como a la tipología de las dimensiones sujetas a evaluación. No obstante, no está exento de las limitaciones propias derivadas de la calidad de la información publicada por las empresas y de su carácter descriptivo. En trabajos futuros sería interesante realizar una descripción de información con indicadores ISO9001; analizar los factores que pudieran explicar las diferencias en la divulgación de información de este tipo de empresas por medio de sus páginas web; detectar la transparencia de las empresas que prestan el servicio en aquellos municipios que no 
son capitales de provincia, así como llevar a cabo el seguimiento de la transparencia en un periodo que abarque varios años de estudio.

A nuestro juicio, prestar atención en materia de transparencia a un sector tan vital para la economía de cualquier región redundará en una mejora de su gestión, y no solo porque puede ayudar a incrementar la confianza de los distintos grupos de interés, sino también para aportar luz sobre si realmente existen diferencias entre la prestación pública o privada de este servicio público.

\section{Referencias}

Alonso, M. L., y García, J. (2014). Evaluación de la transparencia municipal en el Principado de Asturias. Auditoría Pública, 64, 75-86. Recuperado de http:// dialnet.unirioja.es/servlet/articulo? codigo $=4903557$

Anguita, F., Duarte, B., y Flores, S. (2014). Situación actual del transporte público urbano: la visión de las empresas operadoras. Investigaciones Europeas de Dirección y Economía de las Empresas, 20(1), 16-22. doi: https:// doi.org/10.1016/ j.iedee.2013.10.003

Cárcaba, A., y García, J. (2008). Determinantes de la divulgación de información contable a través de Internet por parte de los gobiernos locales. Revista Española de Financiación y Contabilidad, 37(137), 63-84. doi: https://doi.org/10.1080/ 02102412.2008.10779639

Comisión Europea. (2011). Libro Blanco: Hoja de ruta hacia unespacioúnicoeuropeodetransporte:porunapolíticadetransportes competitivay sostenible. Recuperado dehttp://ec.europa. eu/transport/themes/strategies/doc/2011_white_paper/ white-paper-illustrated-brochure_es.pdf.

De Rus, G. (2001). Competencia en transporte público y plazos concesionales en España. Revista del Instituto de Análisis Económico, 2, 255-269. Recuperado de http:// mpra.ub.uni-muenchen.de/12651/

Del Campo, P., Herrador, T. C., y Segovia, A. I. (2015). La transparencia organizativa y económica en la web de las fundaciones: un estudio empírico para España. $R E-$ VESCO. Revista de Estudios Cooperativos. doi: https:// doi.org/ 10.5209/rev_reve.2016.v121.49701

Delgado, M. L., Sánchez, M. A., y Gómez, A. (2013). Financiación del servicio público de transporte urbano: un estudio empírico en las empresas españolas. Investigaciones Europeas de Dirección y Economía de las Empresas, 20(3), 151-162. doi: https://doi.or$\mathrm{g} / 10.1016 / \mathrm{j}$. iedee.2013.05.003
Delgado, M. L., Navarro, E., Mora, L., y Rivero, J.A. (2014). Test de Aplicación municipal de la Ley de Transparencia. Recuperado de http://www.sielocal. com/ estudio/2542/Test-de-Aplicación-municipal-dela-Ley-de-Transparencia

Escamilla, S., Plaza, P., y Flores, S. (2015). Análisis de la divulgación de la información sobre la responsabilidad social corporativa en las empresas de transporte público urbano en España. Revista de Contabilidad, 19(2), 195-203. doi: https://doi.org/10.1016/j. rcsar.2015.05.002

Federación Española de Municipios y Provincias (2009). Informe sobre factores determinantes del transporte público urbano colectivo en España. Madrid: Comisión Ejecutiva de la FEMP. Recuperado de http:// www.femp.es/files/566-637-archivo/Informe\%20 Transporte\%20publico\%20\%20FINAL.pdf.

Haro, A., Sánchez, M. M., y Caba, M. C. (2013). Transparencia de las sociedades de garantía recíproca a través de su web corporativa. Análisis empírico del caso español. Contaduría y Administración, 58, 199-223. doi: https:// doi.org/10.1016/S0186-1042(13)71203-8

INE. (2014). España en cifras 2014. Disponible en http:// www.ine.es/prodyser/ espa_cifras/2014/files/assets/ common/downloads/publication.pdf

Ley 7/1985. Reguladora de las Bases de Régimen Local (RBRL). Abril 2 de 1985. Boletín Oficial del Reino de España número 80 de 3 de abril de 1985.

Ley 16/1987. De ordenación de los transportes terrestres (цотт). Julio 30 de 1987. Boletín Oficial del Reino de España 182 de 31 de julio de 1987.

Ley 19/2013. De transparencia, acceso a la información pública y buen gobierno. Diciembre 9 del 2013. Boletín Oficial del Reino de España número 295 de 10 de diciembre de 2013.

Ley 36/2014. Presupuestos Generales del Estado para el 2015. Diciembre 26 del 2014. Boletín Oficial del Reino de España número 315 de 30 de diciembre de 2014.

Lizcano, J. (2013). El valor de la transparencia en las instituciones públicas y las empresas. Revista de Contabilidad y Dirección, 16, 109-125. Recuperado de http://www.accid.org/revista/documents/El_valor_de_la_transparencia_de_las_instituciones_publicas_y_las_empresas.pdf

Martínez, O., y Barea, P. (2015). ¿Quién paga el transporte urbano y metropolitano en España? ATUC, 74, 42-45. Recuperado de http://www.atuc.es/s ites/default/files/ revista-atuc_74-actualizada.pdf 
Observatorio de Costes y Financiación del Transporte Urbano Colectivo (отUC). (2015). Informe anual del observatorio de costes y financiación del transporte urbano colectivo (2012-2013). Madrid: Instituto de Estudios Económicos.

Ruiz, M. (2013). La financiación del transporte público: propuesta de un modelo basado en Europa (Tesis de doctorado), Universidad de Málaga.
Suárez, H. (2004). Las empresas de transporte de viajeros por carretera: una propuesta de modelo informativo contable externo (Tesis de doctorado), Universidad de Las Palmas de Gran Canaria.

Transparencia Internacional España. (2014). Índice de Transparencia de Ayuntamientos (ITA) 2014. Recuperado de http://transparencia.org.es/ita-2014/ 